\title{
HF Radar Two-Station Baseline Bisector Comparisons of Radial Components
}

\author{
D.P. Atwater and M.L. Heron \\ Marine Geophysical Lab \\ James Cook University \\ Townsville, QLD 4811 Australia \\ Email: daniel.atwater@jcu.edu.au
}

\begin{abstract}
One simple way to evaluate errors in the radial components of surface currents is to compare the values measured from two separate radars along the baseline joining them. This is best done at the midpoint of the baseline where the areas sampled by the radars are equal. This cannot be done if the baseline is close to the coast or over land. Here we compare radial components along the perpendicular bisector of the baseline and show that the rms difference approaches the error as the point of observation approaches the midpoint. The application of this method to SeaSonde data showed $r m s$ differences decreasing until the observations were about $25 \mathrm{~km}$ off shore, and then increasing. We suspect that this increase near the coast is due to a known edge effect in the processing of radials that are derived from calibrated antenna patterns. If we exclude the near-coast data points then the rms differences extrapolate to a value of about $0.10 \mathrm{~m} \mathrm{~s}^{-1}$ at the midpoint of the baseline.
\end{abstract}

\section{INTRODUCTION}

In March 2009 two HF Radar stations in Western Australia were installed and added to the growing Australian Coastal Ocean Radar Network (ACORN) under the federally funded oceanographic monitoring program IMOS (Integrated Marine Observing System). Located at the townships of Seabird and Cervantes (henceforth referred to as SBRD and CRVT, respectively) and separated by a straight line distance of $93 \mathrm{~km}$, the two stations cover roughly $5.0 \times 10^{5} \mathrm{~km}^{2}$ of ocean sea surface (Fig. 1). These two SeaSonde (CODAR Ocean Sensors, Ltd., Mountain View, CA USA) type HF Radar devices operate at roughly $5 \mathrm{MHz}$ with $50 \mathrm{kHz}$ of bandwidth and are positioned to have considerable overlap thereby giving a unique opportunity to monitor and characterise the Leuwin current (a strong, warm, seasonal coastal surface current) as it approaches the Perth submarine canyon.

Various configurations of HF radars routinely observe on time scales between 10 and 180 minutes, and on spatial scales of $7-15$ degrees azimuth and $3-6 \mathrm{~km}$ in range [ [1], [2] ]. Quite an effort has been put into the evaluation of errors for HF radar systems and, although there are good correlations with measurements from other technologies, it remains a challenge to set error bars on the radar data. Self-consistency estimates can be made using the spread of data values in a time series or spectrum and generally lead to errors of around $0.05 \mathrm{~m} \mathrm{~s}^{-1}$ [3], [4], [5], [6] while comparisons between HF radar surface currents and those from other technologies indicate errors of $0.08-0.15 \mathrm{~m} \mathrm{~s}^{-1}$ [7], [8], [3], [9], [10], [11]. Generally this difference is attributed to the limitations of comparing surface currents averaged over an area with those taken at a point.

One method of determining any bias or measuring errors in surface currents from HF radars is to compare currents from two different radars which observe the same part of the ocean [4], [12]. This approach makes use of the fact that a single HF radar station can observe only the component of the surface current in the radial direction from the station to the point of observation on the ocean. If a point is chosen on the baseline which joins the two stations, then the same radial component of velocity is sampled by each station. If the observation is at the midpoint of the baseline, as shown in Fig. 2, then the comparison is made between radial current components which are averaged over similar areas of ocean surface and one can expect an accurate result. If the observation point is closer to one station than the other, then the comparison is between currents averaged over different area sizes and the method suffers the same limitations as comparisons with fixed point measurements, depicted in Fig. 3.

In this paper a new approach is proposed where the differences in radial components are evaluated along the perpendicular bisector of the baseline between the two stations. Variation is also sought as the distances between the observation point and each station change along the baseline, from unequal near one station or the other, to equality at the midpoint of the baseline. Any trend can provide an improved estimate of the difference in radial components at the midpoint of the baseline.

\section{Methods}

An extension of the two station radial component baseline comparison - is to consider the radial components of surface currents to each station along the perpendicular bisector of the baseline as indicated in Fig. 1. In this case the difference between the radial components in general will increase as we go away from the baseline, depending on the direction of the surface current vector. The component of surface current parallel to the baseline is the same for each station and does not contribute to the difference. When computing a baseline comparison, there is a trend in the difference between the observed radial components as the location of the observation approaches the midpoint of the baseline, and at that midpoint the difference is interpreted as bias or error [4]. 
In practice it is sometimes difficult to make observations at different points along the baseline, and this is illustrated in Fig. 2. Along the baseline the areas over which the radial components are averaged sometimes include the coastline. This adds unwanted complexity to the process and casts some doubt on the accuracy of the error estimation. Since radial component measurements from a Doppler radar that are given over land are non-sensical, it is worthwhile to remove them from further consideration.

In addition, the effect of shallow water on the radial measurements has to be considered when excluding the baseline range cells that fall on land [13]. Algorithms and the theory of processing backscatter from HF radar relies on assuming that solutions are obtained from deep water, that is where $d>L / 8$; where $L$ is the wavelength of the Bragg surface gravity waves and $d$ is water depth. It is noted that shallow-water effects begin to play a role when $d=L / 8$. Along the section of coastline in Fig. 1, the depth contour of $20 \mathrm{~m}$ is sufficient to satisfy HF radar limitations. This depth contour, on average, between $S B R D$ and $C R V T$ is roughly $1 \mathrm{~km}$ from the coast. This consideration is important as we begin discussing results in relation to the distance to the baseline between these two stations.

Another important factor to consider when analysing SeaSonde radial components is that those derived from receive antenna calibrations (heretoforth referred to as antenna pattern measurements) add further confusion to radial component baseline comparisons along a straight line coast due to inconsistencies in SeaSonde software processing that tends to favour solutions towards the edge of the pattern [14].

Given that the baseline joining the SBRD and CRVT stations crosses the coastline in many places, it is the evaluation along the perpendicular bisector which may provide the better estimate of error or bias. While the comparisons along the baseline are robust when the baseline is across open water, the use of the perpendicular bisector can give a good estimate of bias or error when the baseline is close to the coast. The currents near to the shore tend to follow the bathymetry contours, which adds to the quality of this estimate. If the surface currents (surface current vectors) are always parallel to the baseline, then the radial components will remain equal to each other as the distance increases along the perpendicular bisector. When the surface currents are approximately aligned with the baseline then the differences between the radial components remains small and the asymptote can be accurately evaluated as we move along the perpendicular bisector towards the baseline.

The approach for setting up the comparisons along the bisector is relatively straightforward and is more about accounting than anything else. Radial measurements from each station that fall within $d R$ (range resolution of each station) of the perpendicular bisector were chosen. At $C R V T$ and $S B R D$ $d R=3.15 \mathrm{~km}$. Then considering points on the bisector that are separated by $d R$ a further search was performed to isolate points that were in a $d R \times d R$ polygon around each point on the bisector, see Fig. 4. Any radial measurements from either

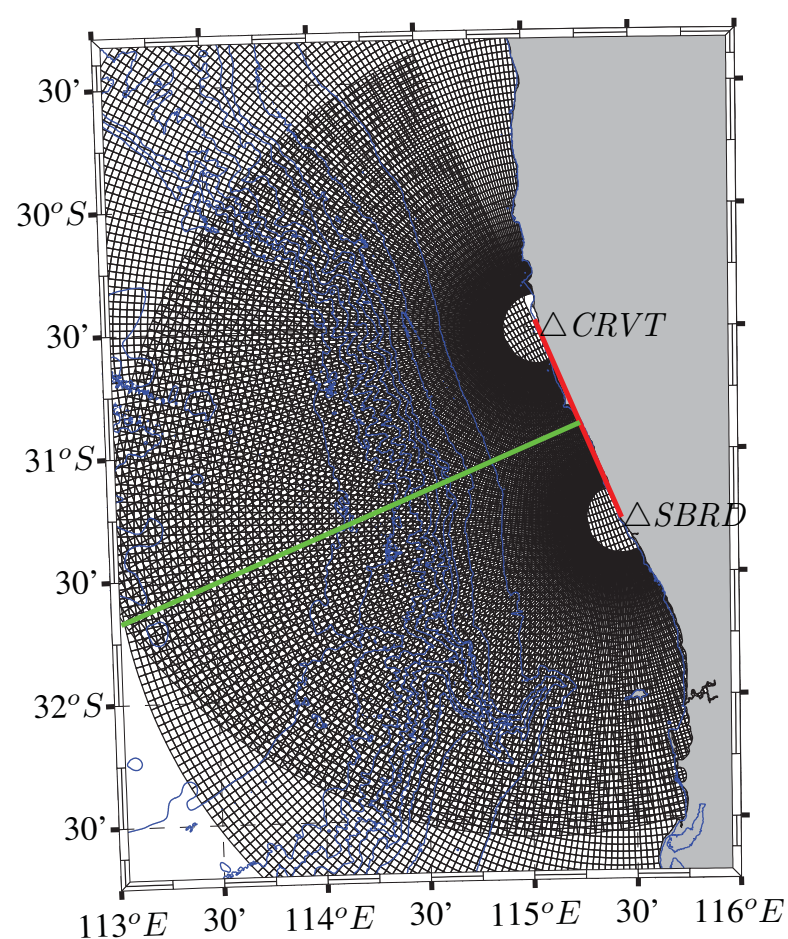

Fig. 1. Map of south central Western Australia coastline, shelf, and Perth submarine canyon. Contours in blue are drawn at 500 meter levels. Baseline between the station is drawn as thick red line and is $93 \mathrm{~km}$ in map length. Notice the proximity of the baseline to the coast. The seaward baseline perpendicular bisector is drawn as a thick green line and is $221 \mathrm{~km}$ in map length.

station that fell on adjoining polygon perimeters were further isolated and chosen to be placed in the westward polygon for consistency purposes.

Beyond this simple narrowing down of radial components to use in the comparison, it is also pertinent to separate radials into a reasonable time range. For this comparison, 01 July 2009 to 30 Sep 2009 was chosen as an adequate sampling period because of the variation in signal quality during this time. A longer time (greater than a month) is found to be necessary so that enough comparisons can be made as to be statistically significant. Since radial components are averaged over 2 hours at each station this made the total number of possible comparisons, at any given location on the perpendicular bisector, 814 .

If $c$ and $s$ represent the 2-hour averaged measured radial components at CRVT and $S B R D$, respectively, and $P_{i}$ are polygons centered at each point on the perpendicular bisector. Then $\bar{c}_{i j}$ and $\bar{s}_{i j}$ are the spatially averaged radial components within each $P_{i}$ for each time sample in the period. So $\bar{c}_{i j}$ and $\bar{s}_{i j}$ are both 81 by 814 matrices of radial component speeds observed along the perpendicular bisector at each time increment. The 81 rows of the matrix represent the perpendicular bisector points seperated by $d R$. 
$31.00^{\circ} \mathrm{S}$

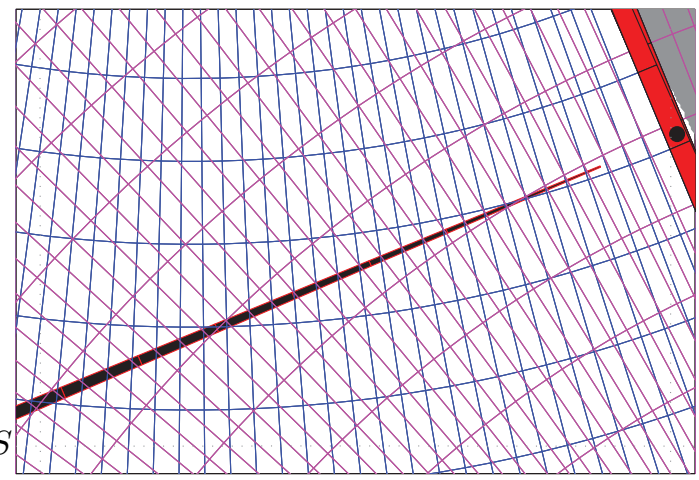

Fig. 2. Map of range cells $S B R D$ (magenta) and CRVT (blue). Red filled range cells represent the baseline cells between each station and the black filled cells represent the bisector of the baseline. The midpoint of the baseline is indicated by the filled black circle.

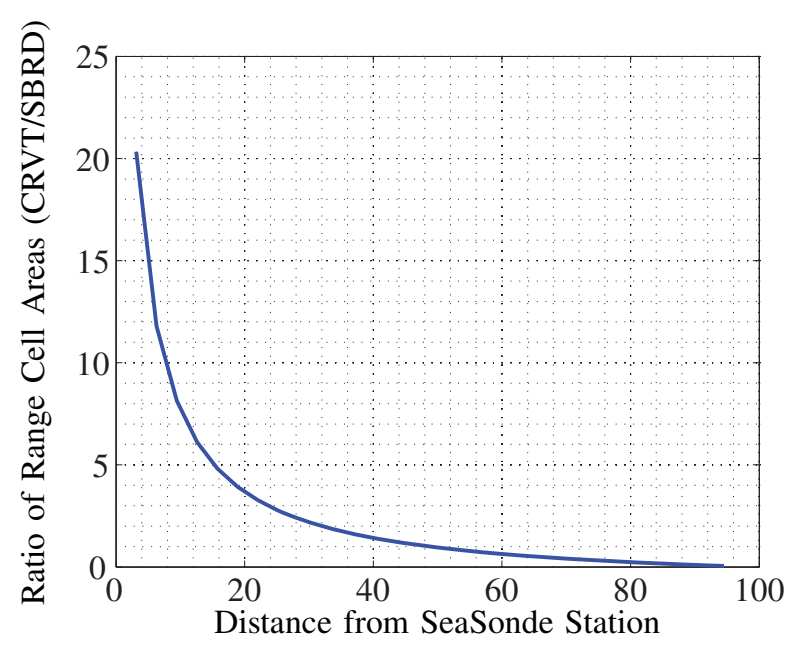

Fig. 3. Shows the simple representation of real range cell area ratios from $C R V T$ and $S B R D$ as a function of distance from the station along the baseline. This illustrates that the distance at which the ratio of the areas from each stations range cell approaches 1 is $46 \mathrm{~km}$, which is the midpoint of baseline between the stations

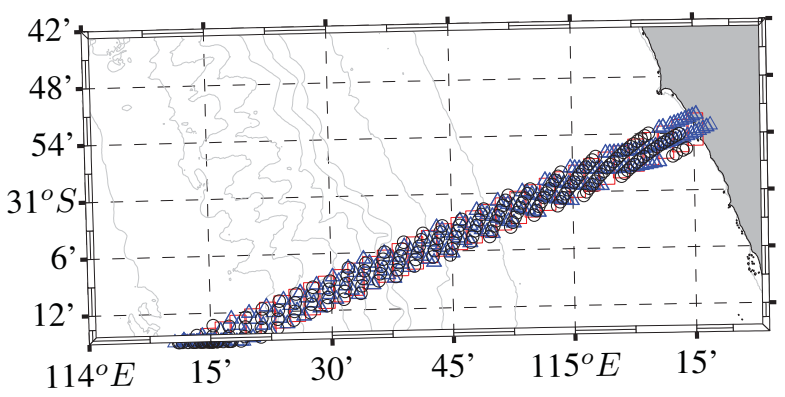

Fig. 4. Mapped section of bisector polygons showing SBRD radial components locations as black circles and $C R V T$ as upward blue triangles. Radial components are a portion of those used in comparison. Ideal and measured radial components from each station do not differ in there location for this study.

\section{RESULTS}

Fig. 5 shows the rms differences $\left(d \bar{S}_{i j}\right)$ between the radial components for the entire time period and supports the intuitive notion that the radial component differences would decrease as the observation point approached the midpoint of the baseline - i.e. the coast.

$$
d S_{i j}=\sqrt{\left(\bar{c}_{i j}-\bar{s}_{i j}\right)^{2}}
$$

and

$$
d \bar{S}_{i}=\frac{1}{N}\left(\sum_{j=1} d S_{j}^{2}\right)^{(1 / 2)}
$$

$\bar{\mu}_{\overline{d S} S_{i}}=0.36 \mathrm{~m} \mathrm{~s}^{-1}$ is the mean of the rms differences over the length of the perpendicular bisector and $\bar{\sigma}_{\overline{d S}}=0.14 \mathrm{~m} \mathrm{~s}^{-1}$ is the mean of the standard deviations of the rms differences over the length of the perpendicular bisector. Fig. 5 shows that the differences reach a maximum of $0.53 \mathrm{~m} \mathrm{~s}^{-1}$ at 50 $\mathrm{km}$ from the coast. A minimum $r m s$ difference of $0.26 \mathrm{~m} \mathrm{~s}^{-1}$ is half as far out from the baseline midpoint at $25 \mathrm{~km}$. This minimum is not as close to the coast as expected and is not as low as other comparisons in the present literature. This discrepancy could be due to a poorly calibrated receive antenna implemented at one of the stations. In particular the antenna calibration is known to cause problems in some radar deployments at bearing angles close to the coastline near the azimuthal extremities of the calibration. Fig. 6 shows antenna pattern measurements for both stations as a polar plot with the magnitude of each cross loop as ratio with the monopole measurement at each bearing. Notice that SBRD's pattern does not extend close to true north and the northern edge of the pattern is at $337^{\circ}$, which is indeed the geographic baseline bearing between the two stations. This is significant in that Cook, et al. have shown that rms differences increase at the edges of antenna pattern measurements [14]. Other than this the patterns do not display any regions that should cause a "piling" of radial measurements, thereby increasing the error. If the calibration is causing the mean rms differences in the radial components to rise near the coast then we might attempt to project the trend observed between $50 \mathrm{~km}$ and $25 \mathrm{~km}$ offshore to estimate the value at the midpoint of the baseline. This would put the rms difference at about $0.10 \mathrm{~m} \mathrm{~s}^{-1}$ at the midpoint of the baseline, which is more consistent with previous measurements of $0.08-0.15 \mathrm{~m} \mathrm{~s}^{-1}$ [7], [8], [3], [9], [10], [11].

\section{CONCLUSION}

From these results it is fair to consider that the $S B R D$ could potentially have a poorly calibrated receive antenna pattern. So a conclusive relative error for these two stations could not be establish in this paper. The next step will be to look at comparing measurements derived from ideal antenna patterns with these results as it would be expected to improve the analysis along the baseline. In essence what has been shown by these results is that radial component differences do decrease approaching the coast along the perpendicular bisector of the baseline, and could be used as measure for determining the 


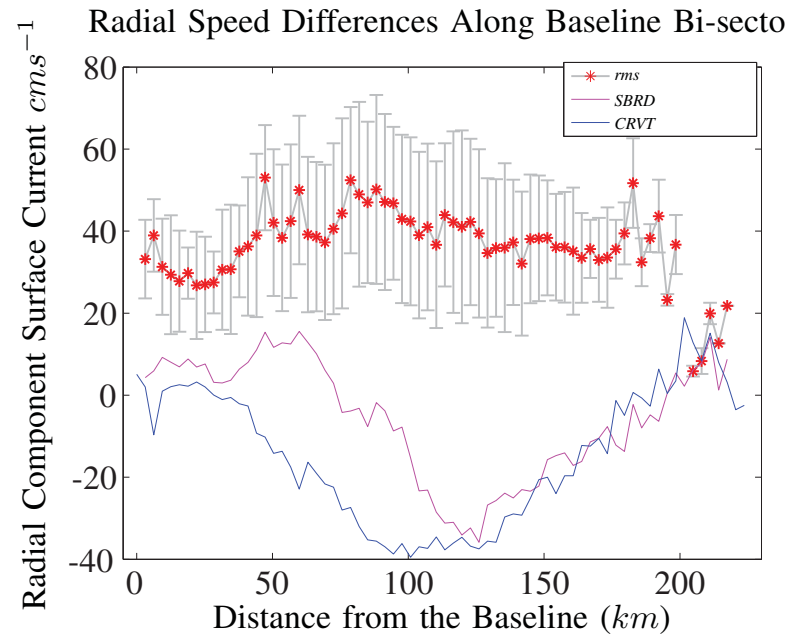

Fig. 5. Radial current components for $S B R D$ and CRVT (solid lines), and simple radial component rms differences as a function of distance from the midpoint of the baseline between the two stations the mean $(\mu)$ and standard deviations $(\sigma)$ over the period 01 July 2009 to 30 Sep. 2009.

relative error of a particular HF radar site. However, it should be noted that this method for comparing radial components not be conducted over short periods as a sufficiently long time period is required to build up enough comparisons as well as allow for significant variations in signal quality. The time range should include a year's worth of radial measurements from both ideal and measured radial patterns. It would also be good to establish a covariance matrix for the radial speeds along the bisector and perform a more sophisticated statistical analysis on the dataset. The aim for this work is to characterise a particular site's (station to station) error bar as a part of setting archival quality control flags for radial components of surface currents.

\section{ACKNOWLEDGEMENTS}

The authors would like to acknowledge data for this work were obtained from the archive: Integrated Marine Observing System (IMOS) at www.imos.org.au . IMOS is part of the Australian Collaborative Research Infrastructure Strategy and the Super Science Initiative.

\section{REFERENCES}

[1] C. Teague, J. Vesecky, and D. Fernandez, "Hf radar instruments, past to present," Oceanography, vol. 10, no. 2, pp. 40-44, 1997.

[2] J. Paduan and H. Graber, "Introduction to high-frequency radar: Reality and myth," Oceanography, vol. 10, no. 2, pp. 36-39, 1997.

[3] H. Essen, K. Gurgel, and T. Schlick, "On the accuracy of current measurements by means of hf radar," IEEE Journal of Oceanic Engineering, vol. 25, no. 4, pp. 472-480, 2000

[4] Y. Yoshikawa, A. Masuda, K. Marubayashi, M. Ishibashi, and A. Okuno, "On the accuracy of hf radar measurement in the tsushima strait," pp. 1-10, 2006.

[5] R. Chapman and H. Graber, "Validation of hf radar measurements," Oceanography, vol. 10, no. 2, pp. 76-79, 1997.

[6] K. Y. D. Toh, "Evaluation of surface current mapping performance by seasonde high frequency radar through simulations," Ph.D. dissertation, Naval Postgraduate School, USA, 2005.

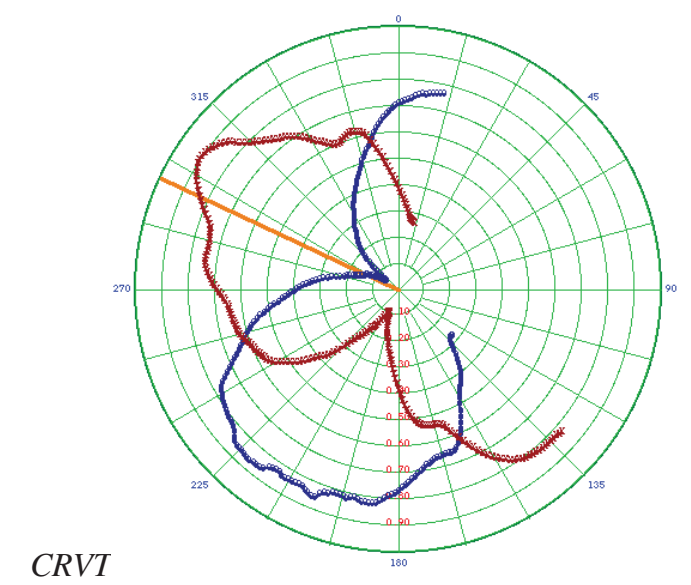

$S B R D$

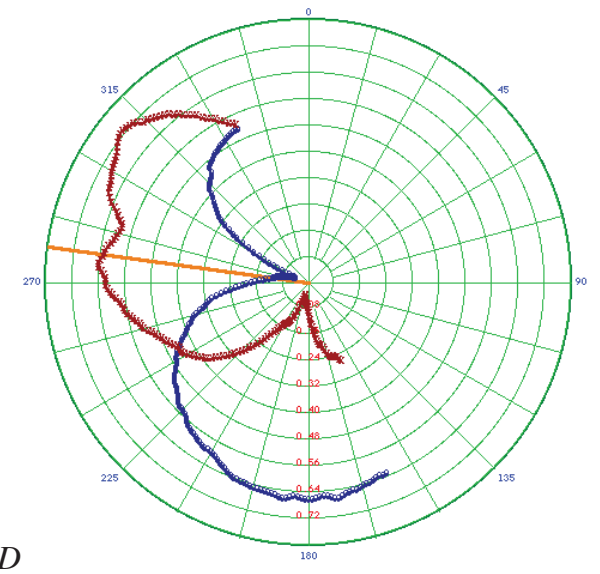

Fig. 6. Receive antenna pattern measurements used at CRVT (top plot) and $S B R D$. Loop 1 is plotted in blue and loop 2 is red. $r$ is the magnitude of the ratio of the signal received at each loop against that of the monopole. $\theta$ are the bearings measred in degrees with true north reference.

[7] G. C. Wright, "Validation of high frequency radar used in ocean surface current mapping via in-situ drifting buoys," Ph.D. dissertation, Naval Postgraduate School, USA, 2008.

[8] J. Paduan, K. Kim, M. Cook, and F. Chavez, "Calibration and validation of direction-finding high-frequency radar ocean surface current observations," IEEE Journal of Oceanic Engineering, vol. 31, no. 4, pp. 862-875, 2006

[9] J. Kohut, H. Roarty, S. Licthenwalner, S. Glenn, D. Barrick, B. Lipa, and A. Allen, "Surface current and wave validation of a nested regional hf radar network in the mid-atlantic bight," IEEE OES CMTC Ninth Working Conference on Current Measurement Technology, pp. 203-207, 2008.

[10] C. Ohlmann, P. White, L. Washburn, E. Terril, B. Emery, and M. Otero, "Interpretation of coastal hf radar-derived surace currents with highresolution drifter data," Journal Atmospheric and Oceanic Technology, vol. 24, pp. 666-680, 2006.

[11] D. A. K. Trujillo, "Accuracy of surface current velocity measurements obtained from hf radar in corpus cristi bay, texas," Ph.D. dissertation, Texas AM University, USA, 2004.

[12] B. Lipa, B. Nyden, D. Ullman, and E. Terrill, "Seasonde radial velocities: derivation and internal consistency," IEEE Journal of Oceanic Engineering, vol. 31, no. 4, 2006.

[13] B. Lipa, B. Nyden, D. Barrick, and J. Kohut, "Hf radar sea-echo from shallow water," Sensors, vol. 8, pp. 4611-4635, 2008.

[14] T. Cook, T. de Paolo, , and E. Terrill, "Estimates of radial current error from high frequency radar using music for bearing determination," IEEE Oceans, vol. Conference Proceedings, 2007. 